\title{
AVALIAÇÃO DA SARCOPENIA EM IDOSOS HOSPITALIZADOS
}

\section{EVALUATION OF HOSPITALIZED ELDERLY SARCOPENIA}

\author{
Nelcimara Lúcia Marafon ${ }^{1 *}$; Bruno Margueritte Costa ${ }^{1}$; Débora Melo Mazzo ${ }^{1}$; \\ Juliana Carvalho Schleder ${ }^{1}$ \\ ${ }^{1}$ Hospital Universitário Regional dos Campos Gerais - HURCG, Ponta Grossa, Paraná, Brasil \\ *Autor correspondente: Rua Pastor Pita 71, apto 12, Uvaranas - Ponta Grossa PR 84030-310 \\ nelci.marafon@gmail.com
}

\section{RESUMO}

A sarcopenia constitui a redução da massa muscular esquelética associada à perda da força muscular e/ou redução do desempenho físico relacionada com o decorrer da idade. A hospitalização e suas consequências são fatores importantes no desenvolvimento ou avançados estágios da sarcopenia. O objetivo desta pesquisa foi determinar o índice de massa muscular esquelética, o desempenho físico e a força muscular e então identificar a sarcopenia de idosos hospitalizados. A amostra foi constituída de 27 participantes divididos em três grupos: grupo de estudo (GE) com idosos hospitalizados $(\mathrm{n}=10)$; grupo controle de adultos hospitalizados (GCA) $(n=9)$ e; grupo controle de idosos de comunidade $(\mathrm{GCI})(\mathrm{n}=8)$. Foram coletados dados antropométricos, realizada avaliação da massa muscular esquelética, da força muscular (preensão palmar) e do desempenho físico (Timed $U p$ and Go). As avaliações foram realizadas em dois momentos com diferença de cinco dias entre a primeira e segunda avaliação. As idosas do GE desenvolveram sarcopenia grave durante a hospitalização com valores finais de TUG 18,33s ( $\mathrm{p}=0,069)$; FPP $6,33 \mathrm{~kg}(\mathrm{p}=0,625)$; IMME $6,34 \mathrm{~kg} / \mathrm{m}^{2}(\mathrm{p}=0,156)$. Os idosos do mesmo grupo apresentaram os seguintes valores: TUG 16,86s $(\mathrm{p}=0,656)$; FPP 21 $\mathrm{kg}(\mathrm{p}=0,741)$; IMME $9,71 \mathrm{~kg} / \mathrm{m}^{2}(\mathrm{p}=0,501)$. Durante o internamento, os idosos do GE mantiveram os valores de força muscular, desempenho físico e índice de massa muscular esquelética dentro dos valores de referências, em contrapartida as idosas do mesmo grupo, desenvolveram sarcopenia grave em cinco dias.

Palavras-chave: Idoso; Hospitalização; Sarcopenia.

\begin{abstract}
Sarcopenia is a reduction of skeletal muscle masses associated with loss of muscle strength and /or reduction of age-related physical performance. Hospitalization and its consequences are important factors in the development or advancement of sarcopenia stages. The purpose of this research to determine skeletal muscle mass index, physical performance and muscle strength and then identify sarcopenia on hospitalized elderly. Sample consisted of 27 participants divided into three groups: study group (SG) with hospitalized elderly $(n=10)$; hospitalized adults group (HAG) $(n=9)$ and; community elderly control group (CEG) $(n=8)$, anthropometric data were collected assessing skeletal muscle mass, muscle strength (palmar gripping) and physical performance (Timed Up and Go test). Evaluations were carried out in two moments with a difference of five days between first and second evaluation. Elderlies of SG developed severe sarcopenia during hospitalization with final values of TUG 18,33 s ( $p=0.069)$; FPP $6.33 \mathrm{~kg}(\mathrm{p}=0.625)$; IMME $6.34 \mathrm{~kg} / \mathrm{m}^{2}(\mathrm{p}=0.156)$. Elderlies from the same group had the following values: TUG $16.86 \mathrm{~s}(\mathrm{p}=0.656)$; FPP $21 \mathrm{~kg}$ ( $\mathrm{p}=0.741)$; IMME $9.71 \mathrm{~kg} /$ $\mathrm{m}^{2}(\mathrm{p}=0.501)$. During hospitalization, elderly of SG maintained values of muscular strength, physical performance and skeletal muscle mass index within the reference values, in contrast, elderly women of the same group developed severe sarcopenia in five days.
\end{abstract}

Keywords: Elderly; Hospitalization; Sarcopenia. 


\section{INTRODUÇÃO}

O envelhecimento é um processo dinâmico associado a diversas modificações que ocorrem nos últimos anos de vida, sejam elas fisiológicas ou morfológicas, que abrangem todos os níveis do organismo. Com o avançar da idade, ocorre à perda ou diminuição da visão e audição, maior propensão à instabilidade postural e imobilidade, aumento da gordura corporal, redução da massa muscular esquelética, entre outras (LEITE et al., 2012).

A sarcopenia é caracterizada pela redução da massa muscular, associada à perda de força muscular, que leva a má qualidade de vida e prejuízos à funcionalidade do idoso (CRUZ-JENTOFT et al., 2010). Diversos mecanismos estão envolvidos na sua etiologia, tais como redução da função neuromuscular, inflamação, estresse oxidativo, alteração na síntese de proteínas, presença de doenças e comorbidades e anormalidades metabólicas e nutricionais (BEAUDART et al., 2014; DIZ et al., 2015).

Um estudo de revisão de literatura encontrou uma prevalência de sarcopenia no Brasil de mínimo de $8 \%$ e máximo de $58,1 \%$ entre sexo masculino e feminino, nas médias de 66,8 a 75,3 anos de idade (BUSS; CONTE; FRANZ, 2016). O Grupo de Trabalho Europeu sobre Sarcopenia em Idosos (EWGSOP) definiu os estágios de sarcopenia pelos seguintes critérios: (1) índice de massa muscular esquelética baixo, (2) força muscular reduzida e (3) baixo desempenho físico. Estes estágios são divididos em: pré-sarcopenia, onde é obrigatório a presença do critério 1 ; sarcopenia com o critério 1 mais o critério 2 ou 3 e; sarcopenia grave com os três critérios presentes (CRUZ-JENTOFT et al., 2010).

Vários fatores podem levar ao aparecimento dos estágios da sarcopenia ou levar a mudança deste, e um dos fatores é a hospitalização. Esta leva à redução ou perda da autonomia, da qualidade de vida, aumento da fragilidade, compromete a capacidade funcional devido à má nutrição, repouso excessivo e a polifarmácia (PEREIRA et al., 2014; NUNES et al., 2017).

Neste contexto, o presente estudo teve como objetivos determinar o índice de massa muscular esquelética, o desempenho físico e a força muscular e então identificar a sarcopenia de idosos hospitalizados.

\section{MATERIAL E MÉTODO}

Este estudo caracterizou-se como um estudo prospectivo e transversal descritivo. Foi aprovado pelo Comitê de Ética em Pesquisa da Universidade Estadual de Ponta Grossa sob o parecer número 2.896.934/2018.

Os critérios de inclusão foram: idosos com 60 anos ou mais de idade, adultos acima de 18 anos, tempo de internação superior ou igual a cinco dias na clínica médica do Hospital Universitário Regional dos Campos Gerais e que possuíam independência funcional para realizar os testes propostos conforme parâmetros descritos no prontuário eletrônico.

Foram excluídos do estudo os participantes que por quaisquer motivos não conseguiram realizar as avaliações sugeridas, que apresentaram alterações nos sinais vitais, que foram transferidos para outra unidade de internação ou receberam alta hospitalar antes da reavaliação.

A seleção da amostra foi por conveniência. A Figura 1 apresenta o fluxograma de distribuição da amostra que inicialmente foi de 45 pacientes elegíveis e no final ficaram 27 divididos em três grupos. $\mathrm{O}$ primeiro grupo constituído por 10 idosos foi nomeado como grupo de estudo (GE). Para viabilização da análise, foram coletados dados de grupo controle de adultos (GCA) composto por 9 participantes também hospitalizados na mesma instituição afim de comparar se o internamento desenvolve sarcopenia e um grupo de idosos hígidos e ativos de comunidade (GCI) com 8 participantes com a finalidade de verificar se a idade e a mobilidade são fatores que predispõem a sarcopenia. 


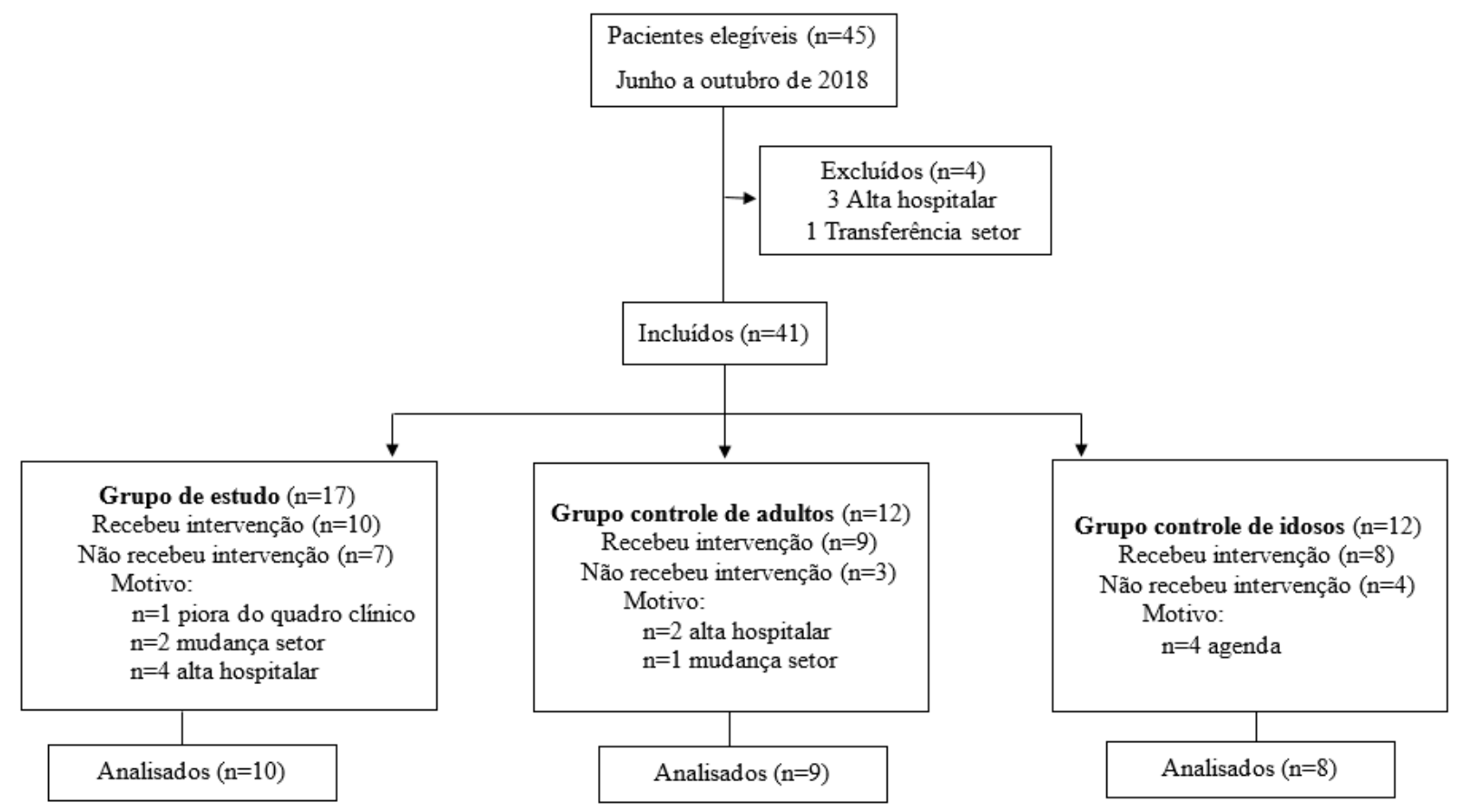

As avaliações dos idosos e adultos internados foram realizadas no hospital, e dos idosos de comunidade foram realizadas no próprio domicílio. Todos os indivíduos foram informados quanto aos procedimentos do estudo e assinaram o Termo de Consentimento Livre e Esclarecido, de acordo com a resolução 466/12.

As avaliações foram realizadas em dois momentos com diferença de cinco dias entre a primeira e a segunda avaliação. Nos pacientes hospitalizados, o tempo máximo para a primeira avaliação era de até 48 horas após o internamento. Inicialmente aplicou-se uma ficha de avaliação contendo os dados sociodemográficos, diagnóstico clínico, comorbidades e medicamentos de uso contínuo.

Posteriormente, os participantes foram submetidos à avaliação antropométrica, mensuração da força de preensão palmar (FPP) e avaliação do desempenho físico. Todas as avaliações foram feitas por uma única pesquisadora. Para aferição da massa corporal, os voluntários foram posicionados em ortostatismo, sem calçados e com os braços ao longo do corpo, em uma balança digital (G-Tech ${ }^{\circledR}$ modelo BALGL10) com capacidade para $150 \mathrm{~kg}$. A estatura foi mensurada com o participante em posição ereta em uma fita métrica fixada na parede. O índice de massa corporal (IMC) foi determinado a partir da divisão da massa corporal em quilogramas pelo quadrado da altura em metros.

Os pontos de corte para a pessoa idosa são IMC $<22 \mathrm{~kg} / \mathrm{m}^{2}$ como baixo peso; IMC entre $22 \mathrm{~kg} / \mathrm{m}^{2}$ e 27 $\mathrm{kg} / \mathrm{m}^{2}$ como eutrófico e IMC $>27 \mathrm{~kg} / \mathrm{m}^{2}$ como sobrepeso (LIPSCHITZ, 1994). Para adultos os valores são IMC $\leq 18,5 \mathrm{~kg} / \mathrm{m}^{2}$ como baixo peso; IMC entre 18,5 $\mathrm{kg} / \mathrm{m}^{2}$ e $25 \mathrm{~kg} / \mathrm{m}^{2}$ como eutrófico; IMC $>25 \mathrm{~kg} / \mathrm{m}^{2} \mathrm{e}$ $<30 \mathrm{~kg} / \mathrm{m}^{2}$ como sobrepeso e IMC acima de $30 \mathrm{~kg} / \mathrm{m}^{2}$ como obesidade (OMS, 2000).

A massa muscular esquelética (MME) foi obtida a partir da fórmula proposta por Lee et al. (2000) que apresenta alta correlação com a densitometria radiológica de dupla energia (RECH et al., 2012) e a ressonância magnética que são exames considerados padrão ouro para esta avaliação. Posteriormente, a MME foi dividida pela altura ao quadrado para obter o índice de massa muscular esquelética (IMME) conforme equação abaixo:

IMME - índice de massa muscular esquelética $\left(\mathrm{kg} / \mathrm{m}^{2}\right)$ $\mathrm{MC}$ - massa corporal $(\mathrm{kg})$

EST - altura (m)

Sexo - homem $=1$ e mulher $=0$

Etnia - 1,2=asiáticos; 1,4=afrodescentes; $0=$ caucasianos.

Como ponto de corte, considerou-se um IMME igual ou inferior a $8,90 \mathrm{~kg} / \mathrm{m}^{2}$ para homens e $6,37 \mathrm{~kg} /$ $\mathrm{m}^{2}$ para mulheres (ALEXANDRE et al., 2014).

Para a avaliação da FPP, avaliada em quilograma (kg), utilizou-se o dinamômetro manual da marca $\mathrm{SGODDE}^{\circledR}$, com capacidade máxima de $130 \mathrm{~kg}$. Os 
participantes foram posicionados sentados em uma cadeira com a coluna ereta, com os pés apoiados no chão, os joelhos fletidos a $90^{\circ}$, ombro aduzido e neutramente rodado, cotovelo fletido a $90^{\circ}$, antebraço em meia pronação e punho em posição neutra, conforme recomenda a American Society of Hand Therapists (ASHT) (DESROSIERS, BRAVO E DUTIL, 1995; FIGUEIREDO et al., 2007).

Coletaram-se três medidas da mão dominante, considerando o maior valor obtido e seguindo os critérios de classificação recomendados pelo Consenso Europeu sobre definição e diagnóstico de sarcopenia que é $<30 \mathrm{~kg}$ para homens e $<20 \mathrm{~kg}$ para mulheres (CRUZ-JENTOFT et al., 2010).

O desempenho físico foi avaliado pelo teste Timed Up and Go (TUG), que consiste em levantar-se de uma cadeira sem ajuda dos braços, caminhar em ritmo confortável e seguro em uma linha reta de três metros, dar a volta, retornar e sentar na cadeira novamente. O participante pôde utilizar calçado e dispositivo auxiliar de marcha, quando necessário. O teste foi cronometrado em segundos. Utilizaram-se para análise do desempenho, os seguintes valores para os idosos: 8, 1s (60 a 69 anos); 9,2s (70 a 79 anos) e 11,3s (80 a 99 anos) (BOHANNON, 2006) e para os adultos adotou-se o valor de $8,1 \mathrm{~s}$.

Após análise da distribuição dos dados da amostra com auxílio do teste de Shapiro-Wilk, foi realizada estatística descritiva para caracterizar as variáveis. Quanto à estatística inferencial para os que apresentarem distribuição normal foram utilizados os testes ANOVA (pós-teste Tukey) e Student t para dados pareados e para os dados não normais, foram utilizados os testes Kruskal-Wallis (pós-teste Dunn) e Wilcoxon para dados pareados.

O nível de significância adotado para os testes estatísticos foi de 5\% em um intervalo de confiança de $95 \%$ e o software utilizado foi o Graph Pad Prism ${ }^{\circledR}$ versão 5.01 para Windows, Graph Pad Software, San Diego Califórnia EUA.

\section{RESULTADOS}

Conforme mostra a Tabela 1 , dos 27 participantes avaliados, quando comparados os três grupos sobre suas características, observou-se que a maioria dos participantes eram do sexo masculino, com média de idade próxima entre os idosos do GE e GCI. Em relação ao IMC, o GE encontrava-se eutrófico, o GCA e o GCI com sobrepeso. Ao analisar o tempo entre as avaliações, todos os grupos apresentaram média de 5 dias, não apresentando diferença estatística $(\mathrm{p}<0,287)$.

Tabela 1: Características da amostra

\begin{tabular}{l|l|l|l|l}
\hline Variáveis & GE $(\mathbf{n}=\mathbf{1 0})$ & GCA $(\mathbf{n}=\mathbf{9})$ & GCI $(\mathbf{n}=\mathbf{8})$ & $\boldsymbol{p}$ \\
\hline Sexo & $\mathrm{F}=3 \mathrm{M}=7$ & $\mathrm{~F}=5 \mathrm{M}=4$ & $\mathrm{~F}=3 \mathrm{M}=5$ & \\
\hline Idade (anos) & $\begin{array}{l}70,20 \\
( \pm 8,90)\end{array}$ & $\begin{array}{l}37,67 \\
( \pm 12,98)\end{array}$ & $\begin{array}{l}68,00 \\
( \pm 8,14)\end{array}$ & $p<0,001^{a}$ \\
\hline IMC $\left(\mathbf{k g} / \mathbf{m}^{2}\right)$ & $\begin{array}{l}25,30 \\
( \pm 5,37)\end{array}$ & $\begin{array}{l}27,29 \\
( \pm 5,56)\end{array}$ & $\begin{array}{l}29,38 \\
( \pm 3,42)\end{array}$ & $\mathrm{p}<0,155^{a}$ \\
\hline $\begin{array}{l}\text { Tempo entre } \\
\text { avaliações } \\
\text { (dias) }\end{array}$ & $5,10( \pm 0,56)$ & $5,00( \pm 0,50)$ & $\begin{array}{l}5,62 \\
( \pm 1,06)\end{array}$ & $\mathrm{p}<0,287^{\mathrm{b}}$ \\
\hline
\end{tabular}

GE-grupo de estudo; GCA-grupo controle de adultos; GCIgrupo controle de idosos; IMC-índice de massa corporal. Valores expressos em média (desvio padrão). Nível de significância $\mathrm{p}<0,05$. aTeste ANOVA de 1 via, pós teste Tukey (diferença entre GE e GCA, GCI e GCA). bTeste Kruskal Wallis.

A Tabela 2 mostra a comparação do desempenho físico, força de preensão palmar e índice de massa muscular esquelética entre os grupos. A variável desempenho físico apresentou diferença estatística significativa entre os grupos em ambas avaliações (inicial $p=0,010$ e final $p=0,013$ ). Não houve diferenças entre as demais variáveis.

Tabela 2: Comparação do desempenho físico, força de preensão palmar e índice de massa muscular esquelética entre grupos.

\begin{tabular}{|c|c|c|c|c|}
\hline & GE & GCA & GCI & p \\
\hline $\begin{array}{l}\text { TUG } \\
\text { inicial (s) }\end{array}$ & $\begin{array}{l}17,00 \\
(14,75-21,75)\end{array}$ & $\begin{array}{l}11,00 \\
(9,00-13,00)\end{array}$ & $\begin{array}{l}11,00 \\
(9,00-15,75)\end{array}$ & $0,010^{\mathrm{a}, \mathrm{c}}$ \\
\hline $\begin{array}{l}\text { FPP inicial } \\
(\mathrm{kg})\end{array}$ & $\begin{array}{l}14,00 \\
(9,75-20,00)\end{array}$ & $\begin{array}{l}26,00 \\
(8,00-72,00)\end{array}$ & $\begin{array}{l}26,00 \\
(10,50-51,25) \\
\end{array}$ & $0,357^{b}$ \\
\hline $\begin{array}{l}\text { IMME } \\
\text { inicial }(\mathrm{kg} / \\
\left.\mathbf{m}^{2}\right)\end{array}$ & $\begin{array}{l}8,61 \\
(8,00-10,68)\end{array}$ & $\begin{array}{l}10,54 \\
(8,34-12,66)\end{array}$ & $\begin{array}{l}9,69 \\
(8,92-10,65)\end{array}$ & $0,209^{\mathrm{a}}$ \\
\hline $\begin{array}{l}\text { TUG final } \\
\text { (s) }\end{array}$ & $\begin{array}{l}15,00 \\
(12,75-22,25)\end{array}$ & $\begin{array}{l}9,00 \\
(7,00-11,00)\end{array}$ & $\begin{array}{l}8,50 \\
(7,25-12,00)\end{array}$ & $0,013^{\mathrm{b}, \mathrm{d}}$ \\
\hline $\begin{array}{l}\text { FPP final } \\
\text { (kg) }\end{array}$ & $\begin{array}{l}13,00 \\
(6,75-18,75)\end{array}$ & $\begin{array}{l}34,00 \\
(9,00-78,00)\end{array}$ & $\begin{array}{l}30,00 \\
(14,75-52,00)\end{array}$ & $0,103^{b}$ \\
\hline $\begin{array}{l}\text { IMME } \\
\text { final }(\mathrm{kg} / \\
\left.\mathbf{m}^{2}\right)\end{array}$ & $\begin{array}{l}8,62 \\
(7,56-10,86)\end{array}$ & $\begin{array}{l}10,99 \\
(7,96-12,69)\end{array}$ & $\begin{array}{l}9,69 \\
(8,74-10,67)\end{array}$ & $0,200^{\mathrm{a}}$ \\
\hline
\end{tabular}

GE-grupo de estudo; GCA-grupo controle de adultos; GCIgrupo controle de idosos; TUG-Timed Up and Go; FPP-força de preensão palmar; IMME-índice de massa muscular esquelética. Valores expressos em mediana (primeiro e terceiro quartil). Nível de significância $\mathrm{p}<0,05$. ${ }^{\mathrm{a}}$ Teste ANOVA de 1 via teste. ${ }^{\mathrm{b}}$ Teste Kruskal Wallis. ${ }^{\mathrm{c} D i f e r e n c ̧ a ~ e n t r e ~ G E ~ e ~ G C A, ~ G E ~ e ~ G C I . ~}{ }^{\mathrm{d}}$ Diferença entre GE e GCA. 
Ao comparar as avaliações inicial e final destes mesmos parâmetros, nota-se que somente no GCA, especificamente para os testes do TUG, houve diferença estatística, conforme mostra a Tabela 3 .

Tabela 3: Comparação da mobilidade, força de preensão palmar e índice de massa muscular esquelética entre as avaliações inicial e final.

\begin{tabular}{ccccc}
\hline & & Inicial & Final & p \\
\hline \multirow{4}{*}{ GE } & TUG $(\mathrm{s})$ & $18,30( \pm 5,79)$ & $17,30( \pm 7,05)$ & $0,573^{\mathrm{a}}$ \\
& FPP $(\mathrm{kg})$ & $17,50( \pm 13,26)$ & $16,60( \pm 14,47)$ & $1,000^{\mathrm{b}}$ \\
& $\mathrm{IMME}\left(\mathrm{kg} / \mathrm{m}^{2}\right)$ & $8,89( \pm 1,90)$ & $8,81( \pm 2,02)$ & $0,466^{\mathrm{a}}$ \\
& TUG $(\mathrm{s})$ & $11,44( \pm 3,20)$ & $9,55( \pm 3,04)$ & $0,007^{\mathrm{a}}$ \\
\multirow{4}{*}{ GCA } & FPP $(\mathrm{kg})$ & $36,78( \pm 33,64)$ & $43,56( \pm 37,97)$ & $0,056^{\mathrm{a}}$ \\
& $\mathrm{IMME}\left(\mathrm{kg} / \mathrm{m}^{2}\right)$ & $10,55( \pm 2,54)$ & $10,55( \pm 2,59)$ & $0,996^{\mathrm{a}}$ \\
& TUG $(\mathrm{s})$ & $12,63( \pm 4,86)$ & $12,13( \pm 9,83)$ & $0,181^{\mathrm{b}}$ \\
\multirow{4}{*}{ GCI } & FPP $(\mathrm{kg})$ & $33,88( \pm 26,70)$ & $39,88( \pm 32,38)$ & $0,177^{\mathrm{a}}$ \\
& $\mathrm{IMME}\left(\mathrm{kg} / \mathrm{m}^{2}\right)$ & $9,64( \pm 1,09)$ & $9,58( \pm 1,13)$ & $0,343^{\mathrm{a}}$ \\
\hline
\end{tabular}

GE-grupo de estudo; GCA-grupo controle de adultos; GCIgrupo controle de idosos; TUG-Timed Up and Go; FPP-força de preensão palmar; IMME-índice de massa muscular esquelética. Valores expressos em média (desvio padrão). Nível de significância $\mathrm{p}<0,05$. aTeste Student t. bTeste Wilcoxon.
Ao avaliar os estágios da sarcopenia, conforme mostram as Tabela 4 e 5, nota-se que as idosas do GE, após avaliação final, encaixam-se nos critérios estabelecidos, classificando-as como sarcopenia grave, pois o IMME está baixo, bem como a FPP e o TUG. $\mathrm{Na}$ avaliação final do GE, em ambos os sexos, houve uma pequena redução no TUG e na FPP, já o IMME dos idosos aumentou. As mulheres do GCA e do GCI, na avaliação final mostraram uma melhora no tempo do TUG e da FPP. Já para os homens do GCI e GCA, somente os valores do TUG estiveram abaixo do valor de referência que é de 8,1s (BOHANNON, 2006).

Tabela 4: Critérios para os estágios da sarcopenia em mulheres.

\begin{tabular}{l|l|l|l|l|l}
\hline & & Inicial & Final & p & Sarcopenia \\
\hline \multirow{4}{*}{ GE } & TUG $(\mathrm{s})$ & $19,00( \pm 9,84)$ & $18,33( \pm 9,23)$ & $0,069^{\mathrm{a}}$ & \\
\cline { 2 - 5 } & FPP $(\mathrm{kg})$ & $7,66( \pm 3,78)$ & $6,33( \pm 0,57)$ & $0,625^{\mathrm{a}}$ & \multirow{2}{*}{ presente (avaliação final) } \\
\cline { 2 - 5 } & IMME $\left(\mathrm{kg} / \mathrm{m}^{2}\right)$ & $7,07( \pm 1,83)$ & $6,34( \pm 1,26)$ & $0,156^{\mathrm{a}}$ & \\
\hline \multirow{4}{*}{ GCA } & TUG $(\mathrm{s})$ & $12,00( \pm 1,00)$ & $10,00( \pm 1,22)$ & $0,021^{\mathrm{a}}$ & \\
\cline { 2 - 5 } & FPP $(\mathrm{kg})$ & $12,00( \pm 8,12)$ & $17,00( \pm 13,08)$ & $0,319^{\mathrm{a}}$ & \multirow{2}{*}{ ausente } \\
\cline { 2 - 5 } & $\mathrm{IMME}\left(\mathrm{kg} / \mathrm{m}^{2}\right)$ & $9,19( \pm 2,36)$ & $9,14( \pm 2,50)$ & $0,813^{\mathrm{a}}$ & \\
\hline \multirow{4}{*}{ GCI } & TUG $(\mathrm{s})$ & $12,33( \pm 3,05)$ & $8,66( \pm 0,57)$ & $0,127^{\mathrm{a}}$ & \\
\cline { 2 - 5 } & FPP $(\mathrm{kg})$ & $12,00( \pm 10,39)$ & $18,33( \pm 5,13)$ & $0,193^{\mathrm{a}}$ & \multirow{2}{*}{ ausente } \\
\cline { 2 - 5 } & IMME $\left(\mathrm{kg} / \mathrm{m}^{2}\right)$ & $8,81( \pm 1,18)$ & $8,80( \pm 1,16)$ & $0,867^{\mathrm{a}}$ & \\
\hline
\end{tabular}

GE-grupo de estudo; GCA-grupo controle de adultos; GCI-grupo controle de idosos; TUG-Timed Up and Go; FPP-força de preensão palmar; IMME-índice de massa muscular esquelética. Valores expressos em média (desvio padrão). Nível de significância p<0,05. ${ }^{a}$ Teste Student $\mathrm{t}$.

Tabela 5: Critérios para os estágios da sarcopenia em homens.

\begin{tabular}{l|l|l|l|l|l}
\hline & & Inicial & Final & p & Sarcopenia \\
\hline \multirow{4}{*}{ GE } & TUG $(\mathrm{s})$ & $18,00( \pm 4,20)$ & $16,86( \pm 6,64)$ & $0,656^{\mathrm{a}}$ & \\
\cline { 2 - 5 } & FPP $(\mathrm{kg})$ & $21,71( \pm 13,78)$ & $21,00( \pm 15,45)$ & $0,741^{\mathrm{a}}$ & \multirow{2}{*}{ ausente } \\
\cline { 2 - 5 } & IMME $\left(\mathrm{kg} / \mathrm{m}^{2}\right)$ & $9,68( \pm 1,39)$ & $9,71( \pm 1,43)$ & $0,501^{\mathrm{a}}$ & \\
\hline \multirow{4}{*}{ GCA } & TUG $(\mathrm{s})$ & $10,75( \pm 4,99)$ & $9,00( \pm 4,69)$ & $0,034^{a}$ & \\
\cline { 2 - 5 } & FPP $(\mathrm{kg})$ & $67,75( \pm 25,06)$ & $76,75( \pm 31,19)$ & $0,139^{\mathrm{a}}$ & \multirow{3}{*}{ ausente } \\
\cline { 2 - 5 } & $\mathrm{IMME}\left(\mathrm{kg} / \mathrm{m}^{2}\right)$ & $12,25( \pm 1,69)$ & $12,31( \pm 1,46)$ & $0,699^{\mathrm{a}}$ & \\
\hline \multirow{4}{*}{ GCI } & TUG $(\mathrm{s})$ & $12,80( \pm 6,05)$ & $14,20( \pm 12,44)$ & $0,681^{\mathrm{a}}$ & \\
\cline { 2 - 5 } & FPP $(\mathrm{kg})$ & $47,00( \pm 24,88)$ & $52,80( \pm 35,57)$ & $0,292^{\mathrm{a}}$ & \multirow{3}{*}{ ausente } \\
\cline { 2 - 5 } & $\mathrm{IMME}\left(\mathrm{kg} / \mathrm{m}^{2}\right)$ & $10,15( \pm 0,75)$ & $10,04( \pm 0,91)$ & $0,379^{\mathrm{a}}$ & \\
\hline
\end{tabular}

GE-grupo de estudo; GCA-grupo controle de adultos; GCI-grupo controle de idosos; TUG-Timed Up and Go; FPP-força de preensão palmar; IMME-índice de massa muscular esquelética. Valores expressos em média (desvio padrão). Nível de significância $p<0,05$. ${ }^{\text {Teste }}$ Student t. 


\section{DISCUSSÃO}

A sarcopenia é uma condição do envelhecimento, diretamente relacionada com a perda da funcionalidade. Sua prevalência varia de acordo com a idade, acometendo de 10 a $25 \%$ dos idosos com até 70 anos de idade e mais de $40 \%$ acima dos 80 anos (MARZETTI et al., 2010). No presente estudo, a maioria dos idosos avaliados encontravam-se na faixa etária dos 70 anos, como descrito em outros estudos (GOBBO et al., 2012; PINHEIRO et al., 2013; ALEXANDRE et al., 2014; MARTINEZ et al., 2015).

A idade é considerada um fator de risco para o desenvolvimento da sarcopenia, principalmente nos idosos acima de 80 anos (DIZ et al.,2015; DUTRA et al., 2015; PONGPIPATPAIBOON et al., 2018). Um estudo evidenciou sarcopenia de acordo com grupos etários, sendo que ela está presente em $25 \%$ dos idosos com 60-69 anos, 33\% dos idosos com 7079 anos, e $60,7 \%$ dos idosos com mais de 80 anos (PONGPIPATPAIBOON et al., 2018).

O desempenho físico, inicial e final, do GCA foi melhor quando comparado com os idosos do GE e GCI. Estes resultados podem ter relação com o melhor entendimento do teste por parte dos adultos, uma vez que o envelhecimento pode comprometer a função cognitiva do idoso acarretando prejuízos (CRUZ et al., 2015) e o processo de aprendizagem é mais facilitado nos adultos quando comparado aos idosos (RODRIGUES; YAMASHITA; CHIAPPETTA; 2008). Ademais, apesar dos valores do TUG do GE terem reduzido na avaliação final, continuaram acima do valor de referência. Fato que pode estar relacionado ao processo de envelhecimento, inclusive ocorre concomitantemente com a redução da força motora, o que influencia no padrão físico desta população (FALSARELLA et al., 2015; MARTINEZ et al., 2016).

A respeito dos valores de TUG dos três grupos, observou-se pequena melhora da avaliação final quando comparado com a inicial, porém nos três grupos os resultados estiveram acima do valor de referência definido por Bohannon (2006). Estudos semelhantes também têm apresentado valores parecidos acima de $8,1 \mathrm{~s}$ para os idosos hospitalizados (MARTINEZ et al., 2015; LOOIJAARD et al., 2018; SIM et al., 2018; RODRIGUES et al., 2018). Assim como Looijaard et al. (2018) que mostrou a média do TUG de 16,6s em 135 idosos de ambos os sexos com média de idade de 80,9 anos. Rodrigues et al., (2018) em seu estudo com 384 idosos de ambos os sexos, relatou que 223 apresentaram TUG de 10 a 20 segundos, e 21 apresentaram TUG acima de 20 segundos. Outro estudo com 201 idosos hospitalizados, entre homens e mulheres mostrou um valor geral de TUG de 19,6s (ANTUNES et al., 2016).

Ainda avaliando o TUG, IMME e FPP nas avaliações inicial e final, pode-se constatar um valor de TUG final acima do valor de referência no GCI. Tratando-se de idosos da comunidade, um estudo que avaliou idosos de ambos os sexos obteve valores de 8,7s a 11,5s (PAULA et al., 2016), corroborando com a presente pesquisa. Outro estudo encontrou tempos de $11,24 \mathrm{~s}$ a $14,58 \mathrm{~s}$ nos idosos do sexo masculino e feminino da comunidade de 60 a 89 anos (AVEIRO et al., 2012), equiparando-se ao valor de TUG encontrado neste estudo.

Ao avaliar o valor do TUG final no GCA, percebeu-se que houve uma melhora de aproximadamente 2s ao comparar a avaliação inicial e final, o que não pode ser observado nos outros grupos. Esse resultado pode ser explicado pelo fato do GCA ser composto por adultos, que tendem a ter uma melhora mais rápida nas condições de saúde do que pessoas idosas.

Em relação aos critérios para os estágios da sarcopenia nas idosas do GE, percebe-se que apresentaram resultados inferiores aos valores de referência conforme a literatura (BOHANNON, 2006; CRUZJENTOFT et al., 2010; ALEXANDRE et al., 2014) nos três critérios. Em relação ao IMME, as idosas do grupo GE diminuíram $0,74 \mathrm{~kg} / \mathrm{m}^{2}$ no valor na avaliação final, o TUG reduziu $0,67 \mathrm{~s}$ e a FPP diminuiu $1,33 \mathrm{~kg}$, valores que as diagnosticaram com sarcopenia grave após cinco dias. O estudo de Martinez et al. (2016) obteve $21 \%$ de sarcopenia em idosos hospitalizados pelo período de 1 a 5 dias. A literatura tem mostrado maior prevalência da sarcopenia em mulheres idosas (YUKI; ANDO; SHIMOKATA, 2014; ALEXANDRE et al., 2014; DIZ et al., 2015). Alguns fatores podem justificar a prevalência de sarcopenia em mulheres, uma delas é que pessoas do sexo feminino possuem menos massa magra que os homens, o que predispõe à sarcopenia. Além disso, a perda da massa muscular não ocorre de forma igual entre os sexos, sendo que as mulheres sofrem uma perda abrupta ao chegar na menopausa (DIAGO; MEJIA, 2013; FILIPPIN et al., 2017). Além de menor massa magra, as mulheres têm 
menor força e menor desempenho físico quando comparado aos homens (YUKI et al., 2015).

Já em relação aos critérios para os estágios da sarcopenia nos idosos do GE, na avaliação final apresentaram redução dos valores do TUG e da FPP, contudo permaneceram fora dos pontos de corte. Em contrapartida apresentaram um discreto aumento na IMME, contudo não foi significativo. Esse dado pode ter sido gerado devido aos idosos estarem com um aporte maior de dieta (BORREGO; CANTARIA, 2013), com hidratação em excesso (AUGUSTO, 1993) ou edema (SMOLINER; CORNEL; RAINER, 2018) no momento da reavaliação. Porém, os idosos do grupo GE não apresentaram sarcopenia.

Quando comparados os resultados do TUG, da FPP e o IMME nos critérios para os estágios da sarcopenia do sexo feminino e do sexo masculino, entre o GE e o GCI, nota-se que os valores do GCI são mais altos. Isso pode ocorrer porque o idoso, quando hospitalizado, tende a apresentar redução da capacidade funcional, mudança na qualidade de vida e consequente aumento do nível de dependência de cuidados, o que favorece a perda da massa muscular pela mobilidade reduzida (OSUNA-POZO et al., 2014; CARVALHO et al., 2018).

Este estudo apresentou algumas limitações, como a dificuldade para conseguir participantes que se enquadrassem dentro dos critérios de seleção no período proposto para as coletas de dados, o que contribuiu com a pequena amostra, dificultando a análise estatística, e por fim restringiu a obtenção de resultados que pudessem ser estendidos para fora da população desta pesquisa. A amostra restrita também implicou na heterogeneidade dos participantes quanto às características antropométricas da amostra. Também não foram analisadas as comorbidades, motivo do internamento, nível de escolaridade e hábitos de vida, como tabagismo e prática de atividade física, fatores estes que poderiam influenciar nos resultados encontrados.

\section{CONSIDERAÇÕES FINAIS}

Pode-se observar, nas avaliações inicial e final, que o IMME dos idosos hospitalizados esteve dentro dos valores de referência, já o TUG e FPP apresentaram valores abaixo do esperado. Nas idosas hospitalizadas, o IMME na primeira avaliação demonstrou valor acima da referência, porém na avaliação final o valor encontrado foi inferior. Para o TUG e FPP, em ambas as avaliações, os valores estiveram abaixo do ponto de corte. Após cinco dias hospitalizados, os idosos do GE não desenvolveram sarcopenia, contudo, as idosas deste mesmo grupo evoluíram para sarcopenia grave.

A literatura sobre a sarcopenia na prática clínica pode ser mais estudada a fim de maior evidência científica. Portanto, sugere-se a realização de novos estudos com um número maior de participantes homogêneos e a análise de fatores que podem vir a interferir no diagnóstico de sarcopenia.

\section{REFERÊNCIAS}

ALEXANDRE, T. D. S. et al. Prevalence and associated factors of sarcopenia among elderly in Brazil: Findings from the SABE study. Journal of Nutrition, Health and Aging, v. 18, n. 3, p. 284-290, 2014.doi:10.1007/s12603013-0413-0

ALEXANDRE, T.S. et al. Sarcopenia according to the European Working Group on Sarcopenia in Older People (EWGSOP) versus dynapenia as a risk factor for mortality in the elderly. J. Nutr. Health Aging, v. 8, n.1, p. 751-760, 2014.doi:10.1007/s12603-014-0450-3

ANTUNES, A. C. et al. Sarcopenia and hospitalisation costs in older adults: a cross-sectional study. Nutrition\&Dietetics, v.74, n. 1, p. 46-50, 2016.doi:10.1111/1747-0080.12287

AUGUSTO, A.L.P. Peso teórico e necessidades energéticas dos indivíduos. Terapia nutricional. São Paulo, p. 21-22, 1993.

AVEIRO, M.C. et al. Mobilidade e risco de quedas de população idosa da comunidade de São Carlos. Ciênc. Saúde coletiva, v.17, n.9, p. 2481-2488, 2012.doi:10.1590/ s1413-81232012000900028

BEAUDART, C. et al. Estimation of sarcopenia prevalence using various assessment tools. Experimental Gerontology, v. 61, p. 31-37, 2015.doi:10.1016/j.exger.2014.11.014

BOHANNON, R. W. Reference Values for the Timed Up and Go Test: A Descriptive Meta-Analysis. Journal of Geriatric Physical Therapy, v. 29, n. 2, p. 64-68, 2006. doi:10.1519/00139143-200608000-00004

BORREGO, C.C.H; CANTARIA, J.S. Efeito da utilização de complemento alimentar em idosos atendidos em um ambulatório na cidade de São Paulo. Rev. Bras. Geriatr. Gerontol. v.16, n. 2, 2013.doi:10.1590/s180998232013000200009

BUSS, V; CONTE, F.A; FRANZ, L.B.B. Prevalência de sarcopenia em idosos no Brasil: uma revisão de literatura. XXIV Seminário de Iniciação Científica, 2016. Disponível 
em: $<$ https://www.publicacoeseventos.unijui.edu.br/index. $\mathrm{php} /$ salaoconhecimento/article/view/6677/5448>. Acesso em: 25 nov. 2018.

CARVALHO, T.C. et al. Impact of hospitalization on the functional capacity of theelderly: A cohort study. Rev. Bras. Geriatr. Gerontol. v.21, n. 2, 2018.doi:10.1590/198122562018021.170143

CRUZ, D. T. et al. Associação entre capacidade cognitiva e ocorrência de quedas em idosos. Cad. Saúde Colet., v. 23, n. 4, p. 386-393, 2015. Disponível em: <http://www.scielo. br/pdf/cadsc/v23n4/1414-462X-cadsc-23-4-386.pdf $>$. Acesso em: 23 out. 2018.

CRUZ-JENTOFT, A. J. et al. Sarcopenia: European consensus on definition and diagnosis. Age and Ageing, v. 39, n. 4, p. 412-423, 2010. doi: 10.1093/ageing/afq034

DESROSIERS, J; BRAVO, G; DUTIL, E. Normative data for grip strength of elderly men and women. The American Journal of Occupational Therapy, v. 49, n. 7, p. 637-644, 1995. Disponível em: <https://www.ncbi.nlm.nih.gov/ pubmed/7573334>. Acesso em: 23 out. 2018.

DIAGO, P.A.O; MEJIA, M.M. La importância Del ejercicio físico para El tratamiento de La sarcopénia. 2013. TCC (Licenciatura em Educación Física y Esportes) - Universidaddel Vale, Colombia, 2013. Disponível em: <http://bibliotecadigital.univalle.edu.co/ bitstream/10893/6835/1/CD-0430813.pdf $>$. Acesso em 24 out. 2018.

DIZ, J. B. M. et al. Prevalência de sarcopenia em idosos: resultados de estudos transversais amplos em diferentes países. Rev. Bras. Geriatr. Gerontol, v. 18, n. 3, p. 665 678, 2015.doi:10.1590/1809-9823.2015.14139

DUTRA, T. et al. Prevalence and factors associated with sarcopenia in elderly women living in the community. Rev Bras Cineantropom Desempenho Hum, v. 17, n.4, p. 460-471, 2015.Disponívelem: <http://www.scielo.br/scielo. php?script=sci_arttext\&pid=S1980-00372015000400460 > . Acessoem: 13 ago. 2017.

FALSARELLA, G.R. et al. Influence of muscle mass and bone mass on the mobility of elderly womem: an observational study. BMC Geriatrics, v. 14, n.13, p. 1-7, 2014.doi:10.1186/1471-2318-14-13

FIGUEIREDO, IM. et al. Teste de força de preensão palmar utilizando dinamômetro Jamar. Acta Fisiatr., v. 14, p. 104110, 2007. Disponível em: <http://www.actafisiatrica.org. br/detalhe_artigo.asp?id=196>. Acesso em: 12 set. 2017.

GOBBO, L. Sarcopenia e dependência para realização das atividades básicas da vida diária de idosos domiciliados no município de São Paulo: Estudo SABE - Saúde, Bem- estar e Envelhecimento (2000 e 2006). 2012. 89 f. Tese (doutorado) - Programa de Pós-graduação Nutrição em Saúde Pública, Universidade de São Paulo, 2012. Disponível em: $<$ http://www.teses.usp.br/teses/disponiveis/6/6138/tde26032012-163220/pt-br.php>. Acesso em: 17 nov. 2018.

LEE, R. et al. Total- body skeletal muscle mass: development and cross-validation of anthropometric prediction models. Am J Clin Nutr., v. 72, n. 3, p. 796-803, 2000. doi:10.1093/ ajen/72.3.796

LEITE, L. E.A. et al. Envelhecimento, estresse oxidativo e sarcopenia: uma abordagem sistêmica. Rev. Bras. Geriatr. Gerontol, v. 15, n. 2, p. 365-380, 2012.doi:10.1590/s180998232012000200018

LIPSCHITZ, D. A. Screening for nutritional status in the elderly. Prim Care, v. 21, p. 55-67, 1994.Disponívelem: $<$ https://www.ncbi.nlm.nih.gov/pubmed/8197257>. Acesso em: 17 ago. 2017.

LOOIJAARD, S. M. L. M. et al. Single physical performance measures cannot identify geriatric outpatients with sarcopenia. The Journal of Frailty \& Aging, v. 7, n. 4, 2018.Disponívelem: <https://www.ncbi.nlm.nih.gov/pmc/ articles/PMC6208736/>. Acesso em: 17 ago. 2017.

MARTINEZ, B. P. et al. Accuracy of the Timed Up and Go test for predicting sarcopenia in elderly hospitalized patients. Clinics, v.70, n.5, 2015.doi:10.6061/clinics/2015(05)11

MARTINEZ, B. P. et al. Existe associação entre massa e força muscular esquelética em idosos hospitalizados? Rev. Bras. Geriatr. Gerontol., v. 19, n. 2, p. 257-264, 2016. doi:10.1590/1809-98232016019.140228

MARZETTI, E. et al. Mitochondrial death effectors: relevance to sarcopenia and disuse muscle atrophy. Biochim Biophys Acta, v.1800, n. 3, p. 235-44, 2010.doi:10.1016/j. bbagen.2009.05.007

NUNES, B. P. et al. Hospitalização em idosos: associação com multimorbidade, atenção básica e plano de saúde. Rev Saúde Pública, v. 51, n. 43, p. 1-10, 2017. Disponível em: $\quad<$ http://www.scielo.br/pdf/rsp/v51/pt_0034-8910rsp-S1518-87872017051006646.pdf >. Acesso em: 14 out. 2018.

OSUNA-POZO, C. M. et al. Review of functional impairment associated with acuteillness in theelderly. Ver Esp Geriatr Gerontol, .v. 49, n. 2, p. 77-89, 2014. Disponível em: <https://www.ncbi.nlm.nih.gov/pubmed/24529877>. Acesso em: 14 out. 2018.

PAULA, J. A. et al. Análise de métodos para detectar sarcopenia em idosas independentes da comunidade. Rev. Bras. Geriatr. Gerontol.,v. 19, n. 2, p. 235-246, 2016. doi:10.1590/1809-98232016019.140233 
PEREIRA, E. E. B. et al. Funcionalidade global de idosos hospitalizados. Rev. Bras. Geriatr. Gerontol, v. 17, n. 1, p. 165-176, 2014. doi:10.1590/s1809-98232014000100016

PINHEIRO, P.A. Instrumentos de triagem para sarcopenia em idosas residentes em comunidade: indicadores antropométricos e testes de desempenho motor. Dissertação (mestrado) - Programa de Pós-graduação em Enfermagem e Saúde, Universidade Estadual do Sudoeste da Bahia, 2013.

PONGPIPATPAIBOON, K. et al. Preliminary study on prevalence and associated factors with sarcopenia in a geriatric hospitalized rehabilitation setting. The Journal of Frailty \& Aging, v. 7, n. 1, 2018. Disponível em: <https:// www.ncbi.nlm.nih.gov/pubmed/29412442>. Acesso em: 14 out. 2018.

RODRIGUES, A.B; YAMASHITA, E.T; CHIAPPETTA, A.L.M. Teste de fluência verbal no adulto e no idoso: verificação da aprendizagem verbal. Rev CEFAC, v.10, n.4, p. 443-451, 2008. Disponível em: <http://www.scielo.br/ scielo.php?pid=S1516-18462008000400004\&script $=$ sci_ abstract\&tlng=pt $>$. Acesso em: 15 out. 2018.

RODRIGUES, R. A. S. et al. Timed up and go test and self-perceived health in elderly: population-based study. Brazilian Journal of Kinanthropometry and Human Performance, v. 20, n. 3, p. 247-257, 2018. doi:10.5007/1980-0037.2018v20n3p247

SIM, M. et al. Utility of four sarcopenia criteria for the prediction of falls-related hospitalization in older Australian women. Osteoporosis International. 2018.doi:10.1007/

s00198-018-4755-7

SMOLINER, C; CORNEL, C.S; RAINER, W. Prevalence of Sarcopenia in Geriatric Hospitalized Patients. JAMDA, v. 15, p. 267-272, 2014.doi:10.1016/s0261-5614(13)600898

WORLD HEALTH ORGANIZATION. Obesity: preventing and managing the global epidemic. Report of a WHO Consultation. World Health Organization Technical Report Series, 894:i-253, 2000.Disponívelem: <https:// www.who.int/nutrition/publications/obesity/WHO_ TRS_894/en/.Acesso em: 03 set. 2018.

YUKI, A. et al. Epidemiology of sarcopenia in elderly Japanese. J Phys Fitness Sports Med, v. 4, n. 1, p. 111-115, 2015.doi:10.7600/jpfsm.4.111

YUKI, A; ANDO, F; SHIMOKATA, H. Transdisciplinary Approach for Sarcopenia. Sarcopenia: definition and the criteria for Asian elderly people. Clin Calcium,v. 24, n. 10, p. 1441-1448, 2014.Disponívelem: <https://www.ncbi.nlm. nih.gov/pubmed/25266088>. Acesso em: 24 nov. 2018. 